\title{
Plant descriptions for engineering tool interoperability
}

\author{
Oscar Carlsson \\ Midroc Automation AB \\ Stockholm, Sweden \\ Email: oscar.carlsson@midroc.se \\ Bilal Ahmad* \\ and Robert Harrison ${ }^{\dagger}$ \\ Automation Systems Group, WMG, the University of Warwick \\ Coventry, United Kingdom \\ Email: b.ahmad@warwick.ac.uk*, robert.harrison@warwick.ac.uk ${ }^{\dagger}$
}

\author{
Daniel Vera \\ Fully Distributed Systems Ltd. \\ Coventry, United Kingdom \\ Email: daniel.vera@fullydistributedsystems.com
}

\author{
Jerker Delsing \\ Luleå University of Technology \\ Luleå, Sweden \\ Email: jerker.delsing@1tu.se
}

\begin{abstract}
The emergence and deployment of connected devices in many domains of application (e.g. industrial production, buildings and facilities, urban environment, etc.) have resulted in the need to achieve integration of multiple and more complex systems. This new environment is stressing the intrinsic limits imposed by monolithic standards, data models and integration methods that focus on specific domains of application, types of systems, or specific aspects of a system.
\end{abstract}

This paper describes the Plant Description Service developed as part of the Arrowhead Interoperability framework (EU ECSEL funded project). The manuscript contains a description of the abstract system descriptive model based on which the Plant Description service was implemented, and describes how the service can be used to achieve integration of several industry standards and data models. One use case and one case study is provided that illustrates how the service was practically implemented to support engineering scenarios in the domain of industrial production. The paper concludes with a critical review of the approach and suggestion for future work and developments.

Keywords-Industrial automation, engineering, interoperability, standards, Arrowhead.

\section{INTRODUCTION}

Large modern projects, such as construction of factories, power plants, airports, or railroad tunnels, incorporates many engineering disciplines and contains a large number of connected devices. In such projects the engineering quickly becomes a complex operation with the need to exchange data between different tools and data sources used by engineers from different disciplines. [1]

In many situations where safety and performance are critical, it is vital that components can be tracked throughout the complete life-cycle, including production, operation, maintenance, re-use and other possible scenarios. Pátkai et al. [2] illustrate this in a case study from the aerospace industry. Using unique identifiers both for the components and for the functional and locational sections that components are associated with lowers the risk of confusion as systems or personnel from different areas or disciplines are required to cooperate and exchange information. With end-to-end engineering, horizontal integration and vertical integration seen as overarching aspects of the German initiative Industrie 4.0 [3], [4] and with Innovative Engineering being seen as one of the main aspects of digital technology, as perceieved by leaders in economy and society according to ITEA-ARTEMIS [5], there is support for more powerful and better integrated engineering tools. In the status report on Reference Architecture Model Industrie 4.0 (RAMI4.0) [4] there is an emphasis on the combination of life cycle aspects, IT representation layers and the traditional automation hierarchies.

For the improvement of engineering tools in the field of industrial automation there is already considerable effort. For the process industry Braaksma et al. [6] have reviewed a large group of standards for asset information, with a focus on collaboration between engineers of different disciplines and information hand-over between different life-cycle phases. For the area of software engineering in industrial automation Vyatkin [7] presents an overview of the current solutions and concepts, where the role of standards such as IEC 61499 [8] and IEC 61850 [9] are highlighted, and the use of modeldriven software engineering in automation is presented as one of the compelling paths for further development in the area. One example of model-driven software engineering that uses structured standards would be the method presented by Pang et al. [10] for how the Piping and Instrumentation Diagrams (P\&ID's) following the standard IEC 62424 [11] can be translated into IEC 61499 Function Blocks (FB's) commonly used in programming automation systems for the process industry.

Himmler et al. [12] have developed a function-based engineering framework, illustrating how a structured and standardized description of a large system can improve the interdisciplinary collaboration throughout the engineering of a plant. As this framework uses a strict functional hierarchy as its basic structure for description of the plant it will present a view that is familiar to many process and automation engineers but if it is the only structure that can provide a good overview of the plant it may become cumbersome to other disciplines such as technicians, maintenance engineers and others that are more concerned with physical hardware. It is to a large degree due to these differences that there may be several different hierarchies 
present in an existing plant. In the worst case scenario there may be several different names for the same object, resulting in confusion and difficulties in communication between different disciplines.

Most approaches to engineering tool interoperability and standardization of engineering data exchange assume that all data to be exchanged will be harmonized around one standard identifying the assets that the data relates to. However, considering the large number of standards that are already in use, often enforced by engineering tools, the likelihood of one standard dominating all engineering data that concerns a large, modern, automated facility is not very high for the near future. To alleviate the situation with several standards at the same site there are already some initiatives on specific synchronization between pairs of complementing standards such as collaboration between Automation ML (IEC 62714) and OPC-UA (IEC 62541) [13] as one example and collaboration between ISO 15926 and Mimosa [14] as another. However, most standards for plant topology propose one primary aspect around which the main hierarchy is formed. In some cases the standards support additional, supplementary hierarchies as well but the main one is usually mandatory and used to order the data in a tree structure for exchange between systems. This is why we propose a simplified solution for describing the different hierarchies and topologies that may exist within the same plant or system of systems, sometimes defined according to different standards or procedures. The Plant description aims to provide a basic common data structure which can be used to refer to different objects in a large system or system of systems and the relations between the objects.

The Plant description services are intended to give a basic common understanding of the layout of the plant or site, providing possibilities for actors with different interests and viewpoints to access their view of the same data-set. In the case of device replacement this is useful for the technician replacing the device to assign which position the new device is in, e.g. which old device it is meant to replace. To provide the option to view the same set of objects in different ways, arranging them in different hierarchies or networks depending on the desired viewpoint the basic data structure is proposed to be based around nodes and links. An example of how a traditional hierarchy could be represented can be seen in Figure 1. Once the objects are identified other tools, services or systems are intended to provide detailed information, based on the object identity provided by the Plant description. Other systems anticipated to provide such information are the systems managing configuration, services for orchestration, systems for meta-data and specialized engineering tools, but there are other possible services as well.

The main benefit of the proposed solution is intended to be a lower risk for misunderstandings between different organizations and disciplines without the need to force all involved parties to implement one standard that works for all purposes. Possible additional benefits include a lower threshold for utilizing engineering and design data from different sources that may be organized according to different standards.

\section{EXISTING STANDARDS AND RELATED WORK}

A number of interesting standards for exchange of engineering data have been identified and discussed. However,

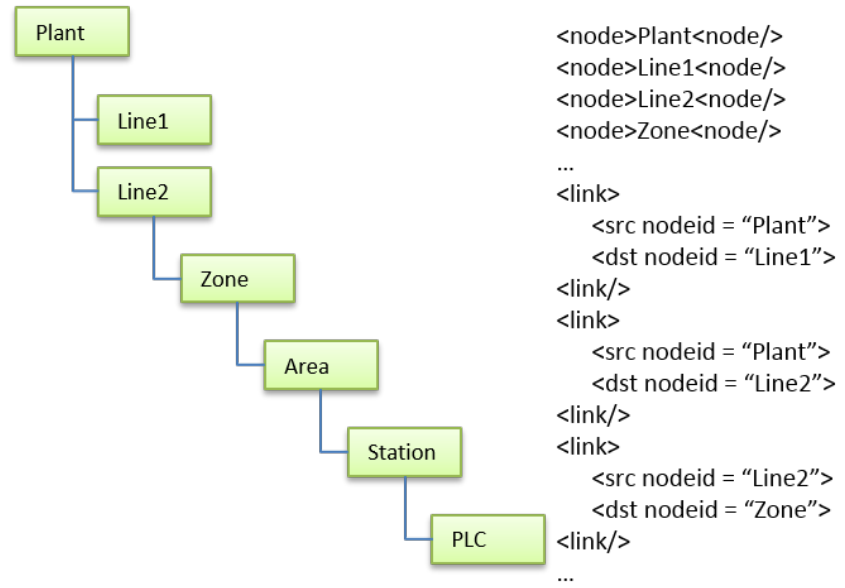

Fig. 1. Hierarchy described by a nodes-and-links data structure

there is no clear solution for the purpose of providing a basis for interaction between engineering tools of the wide spectrum of domains and disciplines covered by the Arrowhead project, including but not limited to production facilities, building automation, infrastructure, electric vehicles and energy systems.

The Reference Architecture Model Industrie 4.0 (RAMI4.0) status report [4], a product of the German initiative Industrie 4.0 , is centered around the standards IEC 62890 for structuring the Life Cycle and Value Stream, combined with the two standards IEC 62264 (ISA-95) and IEC 61512 (ISA-88) for structuring the hierarchical levels. At a more detailed level the report suggests a number of standards for different aspects. For implementation of the Communication layer the report suggests OPC-UA, for the Information layer IEC 61360 (ISO 13584-42), eCl@ss, Electronic Device Description (EDD) and Field Device Tool (FDT) are suggested. Field Device Integration (FDI) is suggested as integration technology and for end-to-end engineering the report suggests ProStep iViP, eCl@ss and AutomationML (which uses a topology based on IEC 62424).

OPC-UA (IEC 62541) [15] is the data exchange standard for platform and vendor independent communication across vertical and horizontal layers within industry in a client-server environment. OPC UA defines generic services and in doing so follows the design paradigm of service-oriented architecture (SOA). In contrast to classic Web services, a number of generic services are already defined and standardized and thus WSDL is not required. Services are organized into logical groupings called service sets. Service requests and response are communicated through message exchange (either using binary protocol on TCP/IP or as a web service) between client and server.

ISO Technical Committee 184 for Automation systems and integration (ISO/TC184) has issued a large number of standards in the field of industrial automation systems [16], including: (in cooperation with IEC) IEC 62264, also know as ISA-95 [17]; ISO 15926 [18] for representation and exchange of life-cycle data of industrial process plants; ISO 10303 for Product data representation and exchange; ISO 15531 for Industrial manufacturing management data. The same technical committee has also released a draft for ISO 18828 that will 
standardize Manufacturing process and management information.

The ISO 15926 standard does allow for multiple disciplines and provides strong support for management of types, classes and instances of objects throughout the complete life-cycle of a process plant. However the ISO 15926 standard is very extensive, as Holm et al. [19] illustrate in the comparison between IEC 62424 and ISO 15926, through the representation of a belt conveyor according to both structures.

The IEC 62264 (ISA-95) is a commonly used standard that defines the hierarchical structure of interaction between an industrial control system and enterprise systems, specifically the functional data flow and object models. However the standard does not in great detail specify the engineering data of the control systems and does not go into the interaction between engineering data of the control system and engineering data of electrical, mechanical or other systems that the control system by necessity are related to.

The ISO/IEC/IEEE standard 15288 [20] specifies a number of concepts that can be useful in the engineering of a large system, while not going into the details of each domain. This standard contains many useful concepts but is not yet widely adopted by the industry and still requires further details for fruitful interoperability between engineering tools of different disciplines.

The standard IEC 81346 describing Industrial systems structuring principles is common for identifying systems and objects in electrical installations within European industries, and is to some extent used within automation systems in such facilities for naming and structuring objects connected to the automation systems. In a similar manner IEC 61850 is used in electrical substation automation systems. The IEC 61850 data model has been mapped to standardized protocol DNP3 (Distributed Network Protocol) [21] for interaction with other automation systems and some interoperability with IEC 61499 has been shown by Yang et al. [22].

The work by Chen and Lin [23] on a Digital Equipment Identifier (DEI) system, which intends to uniquely identify manufacturing equipment and organize data retrieved from vendor Web sites or databases, could be seen as a potential further standard that can be used to describe systems at an automated production site.

As can be seen there are a great number of different standards that in some way concern the modularity of a large production system, by dividing it into objects, functions, locations or systems. To a large extent the modules are likely to be comparable, representing the same physical component, but there may be cases where certain components are neglected as modules for one discipline while very important for another.

\section{ENGINEERING TOOL INTEROPERABILITY}

As with the interoperability of the kind of systems that are the main target of Arrowhead as a whole, the interoperability of engineering tools is possible today as long as all partners follow the same standard or use the same suite of tools from one system provider [24]. However, as the Arrowhead project targets widely different domains [25], including different kinds of production facilities, building automation, infrastructure,

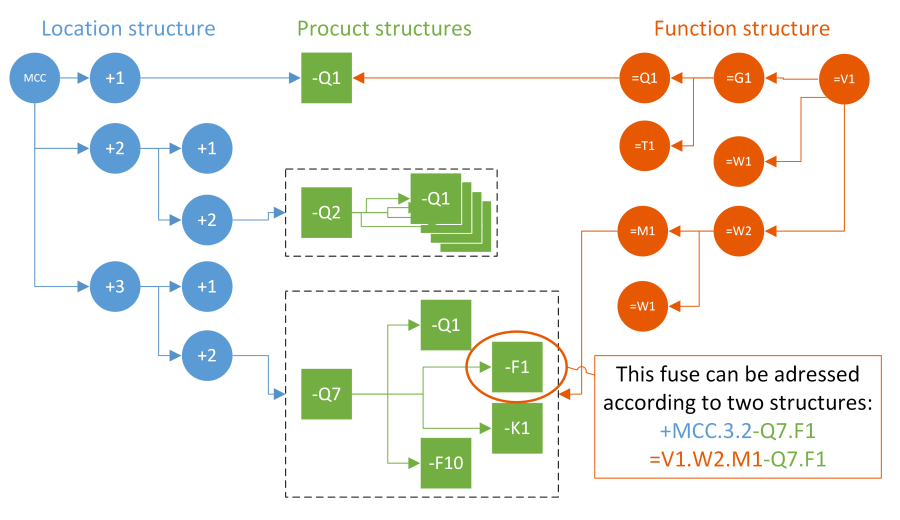

Fig. 2. In IEC81346 the three aspects Function, Location and Product are commonly used to describe different viewpoints of industrial systems

mobile system such as electric vehicles and energy systems; and the engineering tools of those domains have to cover different aspects and life-cycle phases, there are many standards that could be used depending on the domains and lifecycle phase. A first stage of enabling broader interoperability between engineering tools will be to identify a basic form of interaction using services between different tools.

As many domains already follow standards relating to one or more of the aspects that this task aims to address it seems unreasonable to make all of them follow one standard. Instead some inspiration can be taken from the discussions that have already been had within Arrowhead regarding communication protocol translation [26], where the efforts have been concentrated on a solution using one protocol as an intermediary layer and focus on translating to and from this one protocol rather than direct translation between all protocols. Many of the standards concern the parametrization of the object data, which is important for compatibility between tools within one domain, but as the target here is interoperability between tools from different domains it may be sufficient to focus on a few key parameters for each object and the different relations the objects have between themselves.

\section{A. As a service in the Arrowhead Framework}

The purpose of the Plant Description service is to provide a basic common understanding of the layout of a plant or site, providing possibilities for actors with different interests and viewpoints to access their view of the same data set provided by other sources. An source of inspiration for the design was the standard ISO/IEC 81346 which specifies that for studying objects and their relations it may be useful to look at them from different viewpoints, highlighting different aspects of the objects and relations. This standard is focused on the three aspects: function, product and location, although the design is intended to be capable to address other viewpoints as well.

In order to be able to present all of the different types of objects and relations that are expected to be present in a future Internet of Things (IoT) or Industrial Internet of Things (IIoT) network in a useful engineering tool or set of tools the concept of displaying different aspects of the same objects appears to be a useful solution. Figure 2 illustrates how an object documented according to IEC 81346 can be part of a product, visualised in green, addressed either based on its 


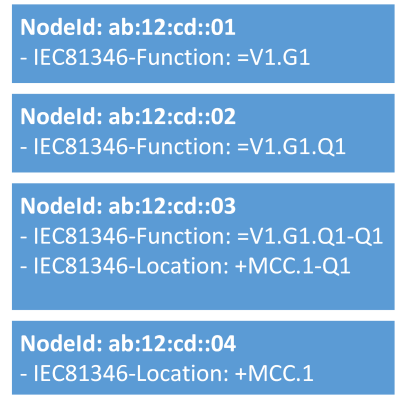

\section{Linkld: cd:12:ff: 78}

Source: Nodeld:ab:12:cd::01

Taurce: Nodeld:ab:12:cd::01

LinkTypes: IEC81346-Function

LinkId: cd:12::ff:79

Source: Nodeld:ab:12:cd:02

Target: Nodeld:ab:12:cd::03

LinkTypes: IEC81346-Function

Linkld: cd:12::ff:7a

Source: Nodeld:ab:12:cd::04

Target: Nodeld:ab:12:cd::03 Target: Nodeid:ab:12:cd::03
LinkTypes: IEC81346-Location

Fig. 3. An example of how the object -Q1 in Figure 2 and three connected nodes could be described by a data structure based on nodes and links.

location, visualised in blue, or the function it performs in the facility, visualised in orange.

\section{B. Nodes and links}

In an effort to limit the amount of data, the proposed solution will only store a few parameter for each object within the Plant description, and a few parameters for each link. Instead, the solution will rely on other data sources, such as existing databases and engineering tools, to provide more specific data.

Therefore the data stored about each object is limited to (1) a unique identifier used within the Plant description and (2) a list of data pairs containing one pair for each standard or other system in which the object is identified and its identifier within that standard and system. Each of these objects is considered a node. Similarly each relation between two objects will be stored as a link, containing four data items: (1) a unique identifier used within the Plant description system, (2) the identifier of the node that is the source of the link, (3) the identifier of the node that is the target of the link and (4) a list of standards and systems in which these two nodes are linked to each other.

Figure 3 illustrates how the nodes $=\mathrm{G} 1,=\mathrm{Q} 1,-\mathrm{Q} 1$ and +1 , and their relations, as found in the top row of Figure 2, can be described by a set of nodes and links.

\section{Plant DESCRIPTION USE CASES}

The Plant description is intended to have multiple usage areas. This section describes one envisioned use case and one case study where a prototype implementation provided some specific required functionality.

\section{A. Design and commissioning}

In a common scenario a group of engineers from different disciplines collaborate to design an automation System of Systems, based on commercially-of-the-shelf (COTS) available automation components, using different engineering tools and standards as appropriate to their respective disciplines. Main requirement is that all engineering tools use a modular approach, identifying components as modules, objects, subsystems or similar, that can be combined into a larger automation system.
The engineers will, throughout the whole process, use the Plant description as a reference tool for objects and systems that have been identified and how they are related to each other. At the early stages the Plant description can help engineers from different disciplines to coordinate their work, even though the design, names of objects, and responsibilities of subsystems may not be fully decided yet.

As engineers from difference disciplines start to populate the design with specific objects, the data can be synchronized between design tools using the Plant description. Throughout the design phase, all of the engineering data is still stored and maintained in the formats preferred by the engineering tools in their respective databases. The data in the Plant description should be limited to what objects exist in the separate databases, how they are identified and what their relations are.

As the systems become ready for commissioning the Plant description can allow technicians to navigate the design using the structure that best fits their knowledge and requirements, while still having the possibility of accessing engineering data from the respective disciplines. Once the systems are commissioned there can be made a direct link between the actual hardware and software on the device and the data from the design and engineering process.

The use of the Plant description should lower the risk of misunderstandings and help identify cases, primarily during design and engineering, where different disciplines use different names for the same object.

\section{B. Automotive industry}

In an effort to implement the concepts and vision of Industrie 4.0, Ford Motor Company, UK is currently undergoing a development phase whose purpose is to achieve integration of the increasing number of interconnected devices deployed in power train assembly plants. In addition to PLC (Programmable Logic Controllers) devices used for control automation, several other types of connected devices are being deployed on the production system itself or linked to various other assets in the shop floor; e.g. RFID tags/antenna systems fitted on material transport and storage (i.e. pallets, racks) for tracking material flow through the shop floor, smart tooling and wearable sensors and trackers, deployment of Resource and Energy Monitors for collecting contextual data ( energy, temperature, vibration). Most devices used are commercialoff-the-shelf products whose deployment and operation rely on specific and often proprietary data structure, connectivity protocols and DBMS (Data Base and Management Systems) back ends. Therefore the design and life-cycle management of future production systems has to focus on the implementation of engineering methods and tools that allow to deal with the increasingly complex nature of CPS.

Figure 4 is a simplified illustration of various data sets describing a same production line from various perspectives which are specific to either engineering domains (e.g. layout, mechanical, control engineering, etc.) or specific to various phases of its life-cycle (e.g. design, commissioning, operation/maintenance). Each views use specific data models (i.e. data types, formats and data structure). For instance, design 


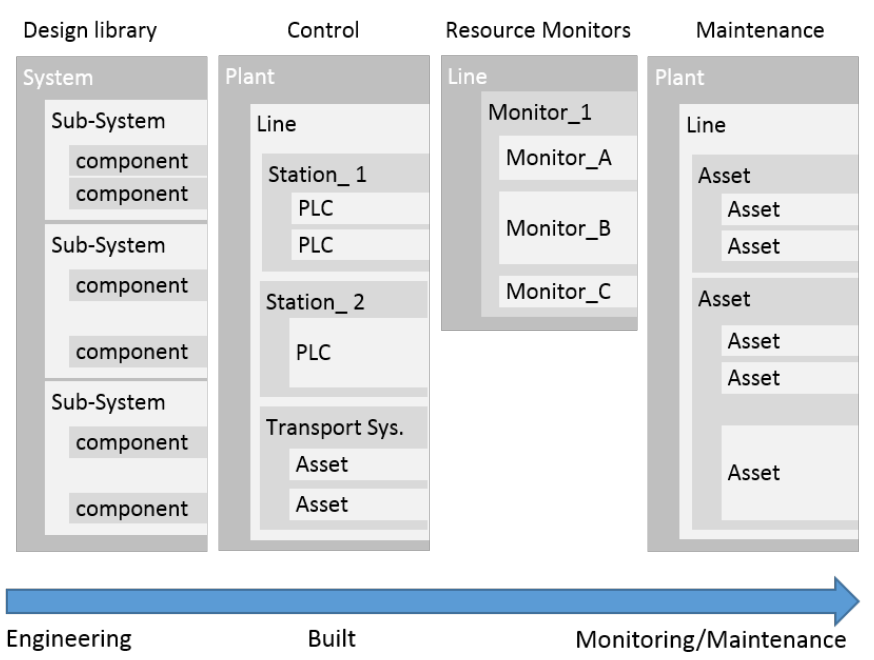

Fig. 4. Four different system hierarchies as used in the automotive industry

libraries (PLM databases) contain product, process and resource (PPR) data used to support digital engineering. Various aspects of the system are typically described at different levels of granularity; For instance a single control PLC may control two or more processing areas as defined in the design library (see Figure 4). Other devices networks such as resource monitoring devices may only be deployed on a sub set of the entire production line so that some aspects of the system are only partially defined compared to others. Heterogeneity at the physical level is reflected in the data models used to store and structure information/data generated by or describing the system. For instance the data structures in both design libraries and maintenance databases (MAXIMO [27] as used by Ford globally) are not consistent as they are targeted at different activities and implemented for different purposes. In addition, the identification of devices which are physically or functionally linked may also be inconsistent: For instance, brass plates and 2D tags are used to uniquely identify physical assets in the shop floor while PLCs and other connected devices typically use IP addresses as unique identifiers. The same assets or devices also exist as a uniquely identified entry in Data Bases.

In this context, the semi-generic descriptive model provided by the Plant Description service was used to a) capture the structure of various sub systems and network of devices that compose complete production lines and facilities and b) map different assets and devices ID using the Nodes and Links element as described in section III-B. This mapping information is essential in enabling navigation and correlation between various data sets and therefore in enhancing cross engineering domains communication and achieve better integration of engineering processes.

The Plant Description model and ID mapping information were used to support the implementation of a so-called Fault Tracker application developed by WMG in the University of Warwick, as described by Harrison et al. [28]. The Fault Tracker mobile application is used in the shop floor in order to support preventative and fault-fixing maintenance of production systems. Figure 5 illustrates how the Fault Tracker is used to aggregate data from different data bases into rich, context

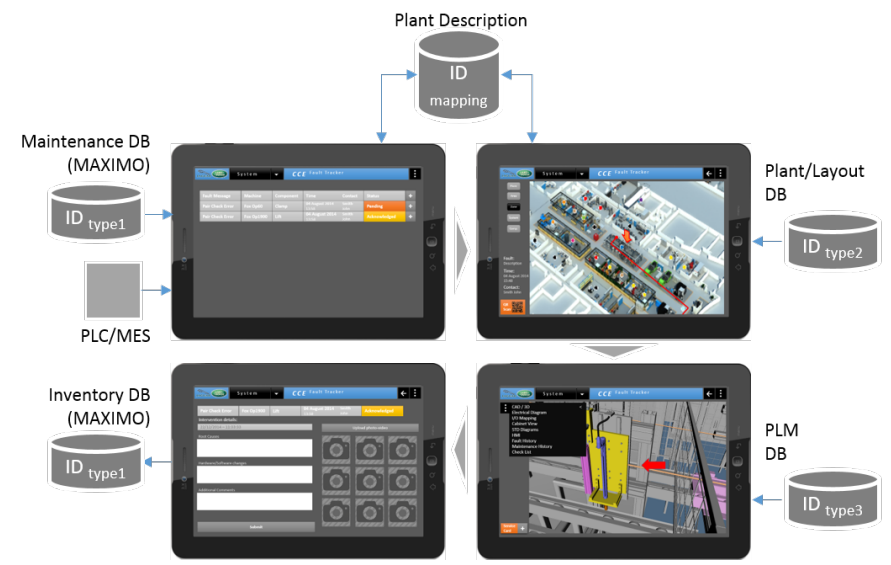

Fig. 5. Fault Tracker maintenance application screens and data sources

sensitive user views supporting various phases of maintenance procedures; a) Fault information and/or maintenance work orders are retrieved from the maintenance database (MAXIMO in this case); based on the maintenance database ID identifying the faulty asset, the b) location of the faulty asset is highlighted in the plant and shop floor layout 2D or 3D CAD data retrieved from the layout engineering database. This information is used to provide location and guidance to the faulty system; c) Views of the faulty component and contextual information are provided (e.g. electrical diagrams, vendor manuals, 3D CAD of faulty component, safety procedures, etc.) in order to assist diagnostic; c) The user can then upload updated maintenance information and/or fault fixing information back to the maintenance database and conduct additional actions such as ordering parts or update the maintenance schedule.

The Fault Tracker case study aims at demonstrating the value of the descriptive model (i.e. Plant Description) overarching several aspects of a same system and of the associated services (e.g. ID mapping) that can be used to identify and retrieve various sets of data and information in order to better support a specific use case (i.e. maintenance of automation systems). The present implementation allows aggregation of maintenance, 2D layout, 3D engineering and control data and presentation into a rich and task specific mobile application user interface. Future implementation will focus on integration of additional data sources e.g. energy monitors, location tracking. Prototype implementation and testing were conducted using the full scale Automation System Workbench demonstration system at WMG, University of Warwick which allowed Ford Motor engineers to identify potential benefits: reduced time to locate and attend asset, reduced time to identify fault, permanent access to up-to date and context/task dependant information, monitoring and traceability of maintenance operations, better management of maintenance personal, paperless work orders management.

\section{CONCLUSION}

The initial purpose of the solution presented in this paper was to provide a simple service interface to navigate different aspects of the standard IEC 81346, most notably to be able to switch between the function aspect and the location aspect, without having to provide all of the detailed data included in each object. 
The solution presented in this paper constitutes an alternative for the exchange of engineering data which does not force all systems to use the same standard or require full compatibility between all relevant standards. The solution provides a basic structure on top of which further compatibility between engineering standards can be developed. Compared to traditional solutions, this solution is more flexible towards the designers of engineering tools but at the cost of adding the additional Plant description data set that needs to be defined and managed. Ideally, the design should be possible to extract from existing engineering data but there is likely some manual identification or matchmaking that needs to be performed as structures from different tools or standards are coordinated.

One aspect of future work would be the identification of objects present in more than one standard to make sure that they are not duplicated in the Plant description. While it is fairly simple to automate the conversion of XML-based topological trees that are used in many standards to nodes and links there may still be the need of considerable engineering effort in synchronizing the nodes created from different standards and to identify possible duplicates.

The future work should also include the design of a full engineering procedure, using the Plant description for integration and coordination. Such a procedure is envisioned to include deployment and programming or configuration of devices into facility, possibly also including management of security features. As a more mature implementation of the Plant description is also part of the future work, this will enable more case studies and more detailed evaluation of the benefits and drawbacks of the approach.

\section{ACKNOWLEDGMENT}

The authors would like to thank all partners of the Arrowhead project for the fruitful discussions and the ARTEMIS JU and ECSEL JU for funding, within project Arrowhead, grant nr. 332987.

\section{REFERENCES}

[1] R. Yang, Process Plant Lifecycle Information Management. AuthorHouse, 2009. [Online]. Available: https://books.google.se/books?id=zmgzdjf1GR0C

[2] B. Pátkai, L. Theodorou, D. McFarlane, and K. Schmidt, "Requirements for rfid-based sensor integration in landing gear monitoring-a case study," Power, vol. 5, p. 4, 2007.

[3] J. H. Henning Kagermann, Wolfgang Wahlster, "Recommendations for implementing the strategic initiative INDUSTRIE 4.0," acatech National Academy of Science and Engineering, Tech. Rep., 2013.

[4] P. Adolphs, H. Bedenbender, D. Dirzus, M. Ehlich, U. Epple, M. Hankel, R. Heidel, M. Hoffmeister, H. Huhle, B. Krcher, H. Koziolek, R. Pichler, S. Pollmeier, A. Walter, B. Waser, and M. Wollschlaeger, "Status Report - Reference Architecture Model Industrie 4.0 (RAMI4.0)," VDI - Verein Deutscher Ingenieure e.V. and ZVEI - German Electrical and Electronic Manufacturers Association, Tech. Rep., July 2015. [Online]. Available: http://www.zvei.org/Downloads/Automation/5305 Publikation GMA Status Report ZVEI Reference Architecture Model.pdf

[5] ITEA and ARTEMIS-IA, "ITEA ARTEMIS-IA High-Level Vision 2030 - Opportunities for Europe," ITEA and ARTEMIS-IA, Tech. Rep., 2013.

[6] A. J. Braaksma, W. W. Klingenberg, and P. P. van Exel, "A review of the use of asset information standards for collaboration in the process industry," Computers in Industry, vol. 62, no. 3, pp. 337 - 350, 2011. [Online]. Available: http://www.sciencedirect.com/science/article/pii/S0166361510001387
[7] V. Vyatkin, "Software engineering in industrial automation: State-ofthe-art review," Industrial Informatics, IEEE Transactions on, vol. 9, no. 3, pp. 1234-1249, Aug 2013.

[8] International Standard IEC61499, Function Blocks, Part 1 - Part 4, IEC Std.

[9] IEC 61850 - Communication Networks and Systems in Substations, IEC Std.

[10] C. Pang, V. Vyatkin, and W. Dai, "IEC 61499 based model-driven process control engineering," in Emerging Technology and Factory Automation (ETFA), 2014 IEEE, Sept 2014, pp. 1-8.

[11] IEC 62424: Representation of process control engineering - Requests in $P \& I$ diagrams and data exchange between P\&ID tools and PCE-CAE tools, IEC Std.

[12] F. Himmler, M. Gepp, J. Vollmar, T. Jager, and M. Amberg, "Functionbased engineering framework for the standardization of industrial plants," in Industrial Electronics Society, IECON 2014 - 40th Annual Conference of the IEEE, Oct 2014, pp. 4909-4915.

[13] M. Schleipen. (2014) Open standards for Industry 4.0 Tools and offer around AutomationML and OPC UA. [Online]. Available: http://www.iosb.fraunhofer.de/servlet/is/46944/AutomationML_en.pdf

[14] A. T. Johnston, "OpenO\&M and ISO 15926 Collaborative Deployment," October 2009. [Online]. Available: http://www.mimosa.org/presentations/openom-and-iso-15926collaborative-deployment

[15] W. Mahnke, S.-H. Leitner, and M. Damm, OPC Unified Architecture. Springer, 2009.

[16] Y. Fukuda, "Standardization for manufacturing systems in iso," in ICCAS-SICE, 2009, Aug 2009, pp. 955-958.

[17] ISA95, Enterprise-Control System Integration, ISA Std.

[18] ISO 15926 Industrial automation systems and integration-Integration of life-cycle data for process plants including oil and gas production facilities, ISO Std.

[19] T. Holm, L. Christiansen, M. Goring, T. Jager, and A. Fay, "Iso 15926 vs. iec 62424 - comparison of plant structure modeling concepts," in Emerging Technologies Factory Automation (ETFA), 2012 IEEE 17th Conference on, Sept 2012, pp. 1-8.

[20] ISO/IEC/IEEE International Standard - Systems and software engineering System life cycle processes, Std., Jan 2008.

[21] "Ieee approved draft standard for exchanging information between networks implementing iec 61850 and ieee std 1815(tm) (distributed network protocol - dnp3)," IEEE P1815.1/D8.00, September 2015, pp. 1-356, Jan 2015.

[22] C.-W. Yang, J. Xu, and V. Vyatkin, "Towards implementation of iec 61850 goose messaging in event-driven iec 61499 environment," in Emerging Technology and Factory Automation (ETFA), 2014 IEEE, Sept 2014, pp. 1-4.

[23] T. Chen and Y.-C. Lin, "A digital equipment identifier system," Journal of Intelligent Manufacturing, pp. 1-11, 2015. [Online]. Available: http://dx.doi.org/10.1007/s10845-015-1071-3

[24] R. Harrison, D. Vera, and B. Ahmad, "Engineering methods and tools for cyber-physical automation systems," 2016, Proceedings of the IEEE, Accepted October 2015 (In Press).

[25] F. Blomstedt, L. Ferreira, M. Klisics, C. Chrysoulas, I. Martinez de Soria, B. Morin, A. Zabasta, J. Eliasson, M. Johansson, and P. Varga, "The arrowhead approach for soa application development and documentation," in Industrial Electronics Society, IECON 2014 - 40th Annual Conference of the IEEE, Oct 2014, pp. 2631-2637.

[26] H. Derhamy, J. Eliasson, J. Delsing, P. Pereira, and P. Varga, "Translation error handling for multi-protocol soa systems," in Emerging Technologies Factory Automation (ETFA), 2015 IEEE 20th Conference on, Sept 2015, pp. 1-8.

[27] IBM. IBM Maximo Asset Management. [Online]. Available: http://www.ibm.com/software/products/en/maximoassetmanagement

[28] R. Harrison, D. Vera, and B. Ahmad, "Engineering methods and tools for cyber-physical automation systems," Proceedings of the IEEE, vol. 104, no. 5, pp. 973-985, May 2016. 